\title{
Coronavirus SARS-CoV-2: Empfehlungen für die nuklearmedizinische Versorgung in nuklearmedizinischen Kliniken/Abteilungen mit Therapiestation und Praxen, Instituten sowie Ordinationen
}

\author{
Deutsche Gesellschaft für Nuklearmedizin (DGN e. V.) \\ und Berufsverband Deutscher Nuklearmediziner (BDN e. V.), in Zusammenarbeit mit \\ der Österreichischen Gesellschaft für Nuklearmedizin und Molekulare Bildgebung (OGNMB)
}

\begin{abstract}
Autoren
Bernd Joachim Krause', Peter Bartenstein ${ }^{2}$, Lutz Stefan Freudenberg ${ }^{3}$, Ken Herrmann ${ }^{4}$, Götz Jonas ${ }^{5}$, Markus Luster ${ }^{6}$, Detlef Mokaㄱ, Wolfgang Schäfer ${ }^{8}$, Sarah Marie Schwarzenböck¹, Philipp Tobias Meyer ${ }^{9}$
\end{abstract}

Institute

1 Klinik und Poliklinik für Nuklearmedizin, Universitätsmedizin Rostock

2 Klinik und Poliklinik für Nuklearmedizin, Ludwig-Maximilians-Universität München

3 ZRN Rheinland und ZRN MVZ GmbH, Korschenbroich

4 Klinik für Nuklearmedizin, Universitätsklinikum Essen

5 Deutsche Gesellschaft für Nuklearmedizin e. V., Göttingen

6 Klinik für Nuklearmedizin, Universitätsklinikum Marburg

7 Zentrum für Nuklearmedizin und Molekulare Bildgebung, Essen

8 Klinik für Nuklearmedizin, Kliniken Maria Hilf GmbH, Mönchengladbach

9 Klinik für Nuklearmedizin, Universitätsklinikum Freiburg
Bibliografie

DOI https://doi.org/10.1055/a-1147-6722

Nuklearmedizin 2020; 59: e1-e4

(C) Georg Thieme Verlag KG, Stuttgart · New York

ISSN 0029-5566

Korrespondenzadresse

Prof. Bernd Joachim Krause

Klinik und Poliklinik für Nuklearmedizin, Universitätsmedizin

Rostock, Gertrudenplatz 1, 18057 Rostock

bernd.krause@med.uni-rostock.de

\section{Einleitung}

Die Coronavirus- bzw. SARS-CoV-2-Pandemie stellt für die medizinische Versorgung eine außerordentliche globale Herausforderung dar. Die Nuklearmedizin ist eine medizinische Disziplin, die neben einem relevanten elektiven Anteil an Diagnostik und Therapie auch in besonderer Weise dringliche bzw. lebenswichtige Untersuchungen und Behandlungen für Patienten* in der Gesundheitsversorgung abbildet. Aufgrund des relevanten wechselseitigen Infektionsrisikos zwischen medizinischem Personal und Patienten sind während der Pandemie dringliche Untersuchungen und Behandlungen gegenüber weniger zeitkritischer elektiver Diagnostik und Therapie zu priorisie-

* $\quad$ Aus Gründen der besseren Lesbarkeit: Personenbezeichnungen gelten gleichwohl für beiderlei Geschlecht. ren. Hierbei sind neben dem Progressions- und Mortalitätsrisiko der Grunderkrankung auch weitere Faktoren zu berücksichtigen (z. B. Nebenwirkungsprofil einer aufschiebenden konservativen Therapie, individuelles Risiko eines Patienten hinsichtlich einer SARS-Cov-2-Infektion bzw. COVID-19, wie z. B. Alter, Komorbidität, Verlassen der häuslichen Isolation, Nutzung öffentlicher Verkehrsmittel etc.).

Grundsätzlich ist festzuhalten, dass die nuklearmedizinischen Einrichtungen durch die Vorgaben des Strahlenschutzgesetzes (Abstand, bauliche Berücksichtigung, Strahlenschutzaspekte für Therapiestationen, Vorgaben bzgl. Handschuhen usw.) und teilweise installierte GMP-Bereiche bereits jetzt eine Reihe von SARS-CoV-2-Maßnahmen in der täglichen Arbeit sehr gut abbilden, was dem Schutz der nuklearmedizinisch behandelten Patienten sowie dem Personal zugutekommt. 
Diese Empfehlung soll eine praktische Hilfestellung geben und Ratschläge anbieten, wie durch weitere z. B. organisatorische Maßnahmen potenzielle Risiken für die Patienten und das Personal minimiert werden können. Diese praktische Handreichung stellt jedoch keine generelle Empfehlung zur etwaigen Reduktion der Leistungserbringung einer nuklearmedizinischen Versorgungsstruktur (nuklearmedizinische Einrichtung mit und ohne Therapiestation, Praxis, MVZ) dar. Diesbezüglich verweisen wir auf die jeweiligen örtlichen Empfehlungen und Anweisungen (z. B. Träger der Institutionen, Ärztekammern, Kassenärztliche Vereinigung oder/oder Gesundheitsämter).

Zu berücksichtigen ist ferner, dass die Versorgungsketten sowohl mit diagnostischen als auch mit therapeutischen Radionukliden instabil werden könnten, bedingt durch die geringe Anzahl von Anbietern/Herstellern bzw. die zum Teil komplizierten Transportwege von der Herstellung bis zum Anwender. Für die Sicherung der diagnostischen Versorgung im Fall einer Lieferkrise kommt den Zentren, die über ein Zyklotron verfügen, eine besondere Bedeutung zu. Dort sollte bereits Vorsorge für den Fall getroffen werden, dass bei einer Unterbrechung der Lieferkette mit diagnostischen Radioisotopen/Radiopharmaka der verstärkte Einsatz von F-18-markierten Radiopharmaka möglich ist. Für die nuklearmedizinischen Therapien, insbesondere im onkologischen Bereich, ergibt sich als Konsequenz, dass sie so lange durchgeführt werden sollten, wie die Versorgung mit therapeutischen Radionukliden und Radiopharmaka gewährleistet ist, und - wenn möglich - nicht verschoben werden sollten.

Übergeordnete Ziele der Empfehlung:

- Schutz von Patienten und Personal

- sowohl vor einer Infektion mit SARS-CoV-2 bzw. einer COVID-19-Erkrankung,

- im Fall der Patienten aber auch vor fehlender/verzögerter Diagnostik und Therapie

- sinnvoller Einsatz von Ressourcen zum Wohle aller

- Solidarität und Hilfe für andere medizinische Disziplinen

\section{DGN- und BDN-Empfehlungen}

\section{Nuklearmedizinische Diagnostik}

Durchführung medizinisch unabdingbarer und notwendiger Untersuchungen, u. a.:

- PET/CT: bei onkologischen Fragestellungen mit unmittelbarem Einfluss auf klinische Entscheidungen und Therapien/Therapieänderungen sowie zur Ermittlung des Therapieansprechens

- Myokardszintigrafie: bei unmittelbarer Relevanz für therapeutische Entscheidungen (s. Appendix 1 „Mögliche technische Untersuchungsmodifikationen ...")

- Wächterlymphknotenszintigrafie als Bestandteil onkologischer Operationen

- Lungenszintigrafie: zum Nachweis und Ausschluss von Lungenarterienembolien (s. Appendix 1 „Mögliche technische Untersuchungsmodifikationen ...“), falls möglich nach Ausschluss COVID-19-typischer pneumonischer Infiltrate durch vorheriges Nativ-CT
- Entzündungsfokussuche bei schwer kranken Patienten (SPECT, SPECT/CT, PET/CT)

- Untersuchungen des Gehirns bei rasch progredienten Erkrankungen (z. B. höhergradige Hirntumoren, Enzephalitiden, ...) und/oder dringlicher Therapieentscheidung

- Skelettszintigrafie auf Basis einer Einzelfallentscheidung

- Schilddrüsenszintigrafie und -sonografie auf Basis von Einzelfallentscheidungen

- Nierenfunktionsszintigrafie auf Basis von Einzelfallentscheidungen

\section{Nuklearmedizinische Therapie}

Nuklearmedizinische Therapien bergen aufgrund der strahlenschutzrechtlichen Regelungen spezifische Probleme, wenn es sich um einen potenziell mit SARS-CoV-2 infizierten Patienten handelt bzw. sich ein Patient im Laufe des stationären Aufenthalts infizieren sollte. Die stationäre Aufnahme von Patienten auf nuklearmedizinische Therapiestationen erfolgt gemäß der Strahlenschutzgesetzgebung bzw. unter strengen Kautelen des Strahlenschutzes, welche bei Verschlechterung des Gesundheitszustands eines Patienten die Verlegung in eine andere Fachabteilung erheblich erschweren und die dortige Ressourcenknappheit verschärfen könnten. Dieses Risiko ist bei evtl. Therapieentscheidungen zu berücksichtigen.

Im Fall der Nutzung von nuklearmedizinischen Therapiestationen als Isolier- und Intensivstationen für die Notfallversorgung im Rahmen der SARS-CoV-2-Pandemie ist eine überregionale (auch länderübergreifende) Sicherstellung von nuklearmedizinischen Zentren zu gewährleisten, die medizinisch unabdingbar indizierte Therapien durchführen.

Sofern es die örtlichen Gegebenheiten und Stationsbelegungen erlauben, sollte die stationäre Versorgung der Patienten aus Gründen des Infektionsschutzes großzügig in Einzelzimmern erfolgen.

Durchführung medizinisch unabdingbarer und notwendiger - onkologischer - ambulanter nuklearmedizinischer Therapien:

- Xofigo ${ }^{\circledR}$-Therapie gemäß zugelassenen Indikationen

Durchführung medizinisch unabdingbarer und notwendiger stationärer nuklearmedizinischer Therapien (idealerweise in Einzelzimmern (oder als Einzelbelegung) mit eigener Nasszelle):

- 1-131-Therapien bei malignen Schilddrüsenerkrankungen in Abhängigkeit vom individuellen Risikoprofil (u. a. Patientenalter und „high risk“-Tumortyp)

- I-131-MIBG-Therapien

- diagnostische I-131-Untersuchungen bei Schilddrüsenkarzinompatienten, die mit dem 4-wöchigen Absetzen der Schilddrüsen-spezifischen Medikation schon begonnen haben

- 1-131-Therapien bei benignen Schilddrüsenerkrankungen in strenger Einzelfallabwägung

- Lu-177-, Ac-225- und Y-90-PSMA-Therapien beim metastasierten kastrationsresistenten Prostatakarzinom nach Ausschöpfung der leitliniengerechten Therapien bei Progress

- Lu-177-/Y-90-Peptid-Rezeptor-Radionuklid-Therapien bei neuroendokrinen Tumoren bzw. SSTR-exprimierenden Tumoren

- Y-90-SIRT (selektive interne Radiotherapie) bei Lebertumoren 
Anzumerken ist ferner, dass im aktuellen Krisenfall in Rücksprache mit den zuständigen Aufsichtsbehörden eine Reduktion der Verweildauer bzw. vorzeitige Entlassung sowie ausgewählte Therapien außerhalb des stationären Settings abzustimmen sind, um Patienten (insbesondere wenn sie in häuslicher Isolation leben) nicht einem unnötigen Infektionsrisiko auszusetzen und Ressourcen maximal zu schonen.

\section{Allgemein}

Idealerweise werden alle Patienten am Vortag der Untersuchung telefonisch kontaktiert und nach Symptomen (Fieber, Husten, Erkältungszeichen) befragt, um eine Präselektion vornehmen zu können - auch wenn eine COVID-19-Infektion asymptomatisch bzw. oligosymptomatisch verlaufen kann. Bei Ankunft in der nuklearmedizinischen Versorgungsstruktur ist ein Screening-Prozess für zugewiesene Patienten (z. B. Kurzfragebögen und Temperaturmessung (wenn auch unspezifisch)) einschließlich Informationserhebung über Kontakte zu nachgewiesen infizierten Personen (gem. Robert-Koch-Institut (RKI)) innerhalb der letzten 14 Tage zu empfehlen. Im Fall auffälliger Befunde ist die kurzfristige Verschiebung der geplanten Behandlung und eine weitere Abklärung der Symptome dringend zu prüfen bzw. eine Virustestung durchzuführen.

Realisierung und Übung entsprechender Verhaltensmaßnahmen innerhalb der nuklearmedizinischen Versorgungsstruktur (Isolation, Schutzmaßnahmen/-ausrüstung des Personals, Desinfektions-/Reinigungsmaßnahmen (einschl. Kamerasysteme u. Ä.) etc.), um im Fall von SARS-CoV-2 positiven Patienten vorbereitet zu sein, die zu medizinisch unabdingbaren Untersuchungen/ Therapien intern zugewiesen werden. Basierend auf den Erfahrungen anderer Länder ist jedoch praktisch nicht mit einer Zuweisung von bekannt SARS-CoV-2-positiven Patienten zu rechnen. Bei der unmittelbaren Versorgung von COVID-19-Patienten kommen der nuklearmedizinischen Diagnostik und Therapie nach aktuellem Kenntnisstand keine relevante Rolle zu.

Grundsätzlich soll enger Kontakt zu Patienten (wie vom RKI definiert) vermieden werden, sofern medizinisch möglich (z. B. genaue sprachliche Instruktion der Patienten bei der Lagerung statt manueller Führung und Positionierung). Bei engem Kontakt und Umlagerung immobiler Patienten ist der Einsatz eines chirurgischen Nasen-Mund-Schutzes zum gegenseitigen Infektionsschutz zu prüfen (s. hierzu auch die jeweils gültigen institutionellen bzw. allgemeinen Empfehlungen, z. B. RKI). Schutzausrüstung und geeignete Mittel zur Händedesinfektion sollten vorgehalten werden. Im Wartebereich ist enger Kontakt der Patienten untereinander durch Einhaltung von Mindestabständen (mindestens $1,5 \mathrm{~m}$ ) bestmöglich zu vermeiden.

Die Durchführung einer Sonografie der Schilddrüse/der Halsweichteile sollte vom Arzt nur unter Verwendung eines NasenMund-Schutzes, ggf. einer Atemschutzmaske, und Handschuhen durchgeführt werden und der Kontakt zum Patienten während der Untersuchung sollte möglichst kurz gehalten werden. Der Ultraschallkopf sollte nach jedem Patienten desinfiziert werden. Zum Schutz des Ultraschallkopfes vor aggressiven Desinfektionsmitteln ist die Verwendung von Schutzhüllen zu empfehlen.
In nuklearmedizinischen Versorgungsstrukturen sollten nach Möglichkeit abgeschlossene Personaleinheiten ohne sog. „faceto-face“-Kontakte etc. gebildet werden, sowohl horizontal (z. B. zwischen Ärzten) als auch hierzu passend vertikal (z. B. zwischen zugeordneten MTRA/RT- oder Pflege-Teams). Diese Empfehlung gilt auch für mittelbar an der Patientenversorgung beteiligte Mitarbeitergruppen, um die Funktionsfähigkeit der Einrichtungen in allen Bereichen auch bei steigenden Infektionsraten aufrechterhalten zu können (d. h. auch im Bereich Anmeldung, Sekretariat, Medizinphysik, Radiopharmazie etc.). Kontaktaufnahmen zwischen Gruppen sollten - wenn möglich - per Telefon oder Videokonferenz erfolgen. Ansonsten sollte ein Mindestabstand von $2 \mathrm{~m}$ - wo immer möglich - eingehalten werden. Schließlich sollten Mitarbeitern großzügig, in Abstimmung mit den zuständigen Organen, Homeoffice-Möglichkeiten bzw. Freistellungen eingeräumt werden (z. B. auch für die Kinderbetreuung während KiTaund Schulschließung); diese Mitarbeiter stellen gleichzeitig Reserve-Teammitglieder bei möglichem erkrankungsbedingtem Personalausfall dar.

Das Personal hat sich entsprechend der RKI-Empfehlungen zu verhalten (Hygienemaßnahmen, Kontakt zu nachgewiesen SARSCoV-2 infizierten Personen). Bei unspezifischen Symptomen/ Krankheitsanzeichen ist bzgl. der Anwesenheit am Arbeitsplatz eher restriktiv vorzugehen (s. aktuelle Empfehlungen des RKI bzw. des zuständigen Gesundheitsamtes). Alle zu ergreifenden Maßnahmen bei Vorliegen einer SARS-CoV-2-Infektion eines Mitarbeiters sollen bekannt sein (entsprechend RKI, Gesundheitsamt bzw. interner Regularien).

Bei der Befundung nuklearmedizinischer Untersuchungen, die eine CT der Lunge umfassen, sollte besonderes Augenmerk auf morphologische Veränderungen der Lunge gelegt werden, die im Zusammenhang mit einer COVID-19-Infektion stehen können $^{3}$. Solche Befunde sollten im Gesamtkontext (Klinik des Patienten etc.) betrachtet und entsprechende Maßnahmen (inkl. Information des Zuweisers) eingeleitet werden.

Ein wichtiger Beitrag, den die Nuklearmedizin in dieser Krise leisten kann, ist die Personalgestellung an andere, mit der akuten Versorgung von COVID-19-Patienten betreute Kliniken und Bereiche. Dies sollte aufgrund des teils relevant reduzierten Leistungsaufkommens großflächig möglich sein. Ärzte, Pflegekräfte und auch andere Mitarbeiter können hierbei z. B. in anderen Abteilungen insbesondere intensivmedizinisch erfahrenere Kollegen entlasten und dazu beitragen, diese Kolleginnen und Kollegen für die extrem aufwendige intensivmedizinische Betreuung von COVID-19-Patienten freizustellen.

Aufgrund der rasanten Entwicklung der aktuellen Krise bedarf das vorliegende Dokument absehbar einer Aktualisierung und kann keinen Anspruch auf Vollständigkeit und Allgemeingültigkeit erheben. Die Autoren werden sich um eine zeitnahe Aktualisierung bemühen. Bitte informieren Sie sich auf der Homepage von DGN und BDN bzw. OGNMB jeweils über den aktuellen Stand und unterstützen Sie uns mit Ihrem Feedback und konstruktiven Vorschlägen. 


\section{Weiterführende Informationen zu diesen Empfehlungen}

Appendix 1: Mögliche technische Untersuchungsmodifikationen zur Anpassung der Workflows an die aktuelle Pandemiesituation mit COVID-19. https://www.nuklearmedizin.de/leistungen/news/ covid.php?navld=234

\section{Weiterführende Informationen zum Coronavirus SARS-CoV-2}

Das Robert-Koch-Institut erfasst kontinuierlich die aktuelle Lage, bewertet alle Informationen, schätzt das Risiko für die Bevölkerung in Deutschland ein und stellt Empfehlungen für die Fachöffentlichkeit zur Verfügung.

Darüber hinaus sind die Erlasse der Bundesländer mit Weisungen an die nachgeordneten Behörden sowie die Vorgaben der Kliniken und Niederlassungen zu beachten.

- Informationen des Robert-Koch-Instituts (RKI) zum Coronavirus SARS-CoV-2 (insbesondere zu Prävention und Bekämpfung im medizinischen Bereich; wird laufend aktualisiert) https://www.rki.de/DE/Content/InfAZ/N/Neuartiges_Corona virus/nCoV_node.html

- Bundesministerium für Gesundheit https://www.bundesgesundheitsministerium.de/coronavirus. html

- Bundeszentrale für gesundheitliche Aufklärung https://www.infektionsschutz.de/coronavirus/

- Deutsche Krankenhausgesellschaft (DKG) https://www.dkgev.de/dkg/coronavirus-fakten-und-infos/

Diese Empfehlungen der Deutschen Gesellschaft für Nuklearmedizin (DGN e. V.) und des Berufsverbands Deutscher Nuklearmediziner (BDN e. V.) stellen den aktuellen Stand des Wissens dar. Die Medizin im Allgemeinen sowie die Erkenntnisse zur aktu- ellen Ausbreitung von SARS-CoV-2 bzw. COVID-19 im Besonderen unterliegen einem fortwährenden Entwicklungsprozess, sodass alle Angaben, insbesondere zu diagnostischen und therapeutischen Verfahren, immer nur dem Wissensstand zum Zeitpunkt der Veröffentlichung dieser Empfehlungen entsprechen können.

Die bereitgestellten Informationen bzw. deren Inhalte wurden nach bestem Wissen und Gewissen erstellt. Die Deutsche Gesellschaft für Nuklearmedizin (DGN e.V.) und der Berufsverband Deutscher Nuklearmediziner (BDN e.V.) sowie die Österreichische Gesellschaft für Nuklearmedizin und Molekulare Bildgebung (OGNMB) übernehmen keine Gewähr für die Aktualität, Vollständigkeit und Richtigkeit der bereitgestellten Informationen bzw. deren Inhalte.

Der Benutzer/behandelnde Arzt selbst bleibt verantwortlich für jede diagnostische und therapeutische Applikation, Medikation und Dosierung.

\section{Interessenkonflikt}

Die Autorinnen/Autoren geben an, dass kein Interessenkonflikt besteht.

Literatur

[1] Conseil National Professionel de Médicine Nucléaire, Version 0.4 en date du 18 mars 2020. Covid-19 - Recommendations portant sur l'organistion des soins en médicine nucléaire.

[2] Huang HL, Allie R, Gnanasegaran G et al. COVID19 - Nuclear Medicine Departments, be prepared! Nuclear Medicine Communications 2020; 41 : 297-299

[3] Qin C, Liu F, Yen TC et al. 18F-FDG PET/CT findings of COVID-19: a series of four highly suspected cases. Eur J Nucl Med Mol Imaging 2020. doi:10.1007/s00259-020-04734-w

[4] Society of Nuclear Medicine \& Molecular Imaging. OVID-19 and Ventilation/Perfusion (V/Q) Lung Studies. http://www.snmmi.org/NewsPublicati ons/NewsDetail.aspx?ItemNumber=33543 (letzter Zugriff: 21.03.2020) 